\title{
Specific matrix metalloproteinases and calcification factors are associated with the vulnerability of human carotid plaque
}

\author{
ZHOU-YING GUO ${ }^{1,2}$, BAI ZHANG $^{1}$, YAN-HONG YAN ${ }^{1}$, SHANG-SHANG GAO $^{3}$, \\ JING-JING LIU ${ }^{3}$, LAN XU ${ }^{3}$ and PIN-JING HUI ${ }^{1}$ \\ ${ }^{1}$ Department of Neurosurgery, The First Affiliated Hospital of Soochow University, Suzhou, Jiangsu 215006; \\ ${ }^{2}$ Department of Ultrasound, The First Hospital of Lianyungang, Lianyungang, Jiangsu 222000; \\ ${ }^{3}$ Department of Biochemical and Molecular of Medical College, Soochow University, Suzhou, Jiangsu 215123, P.R. China
}

Received May 25, 2017; Accepted April 13, 2018

DOI: $10.3892 / \mathrm{etm} .2018 .6424$

\begin{abstract}
The rupture of atherosclerotic plaque provokes the majority of acute cerebrovascular events. Studies have demonstrated that various matrix metalloproteinases (MMPs) may promote atherosclerotic plaque progression and rupture. However, results have been incongruous and the mechanisms of this remain obscured. Therefore, in the current study, carotid plaques were characterized by assessing the levels of MMPs and calcification factors, and evaluating their association with plaque vulnerability. Human carotid plaques were obtained from carotid endarterectomies, and classified into stable and vulnerable groups by ultrasonography and histological analyses. The mRNA and protein levels of MMPs, vascular endothelial growth factor (VEGF), bone sialoprotein 2 (BSP) and osteopontin were investigated by reverse transcription-quantitative polymerase chain reaction and western blotting, respectively. Immunohistochemistry was used to localize MMP-2 and MMP-14 in stable and vulnerable plaques. The activation of various associated signaling pathways was also investigated using western blotting. The mRNA levels of MMP-2, -7, -9 and -14 were elevated in vulnerable plaques, among which expression of MMP-2 and -14 were the highest. Consistent with the mRNA levels, the protein levels of MMP-2 and -14 were also elevated. Immunohistochemistry also demonstrated positive staining of MMP-2 and MMP-14 in vulnerable plaques. Factors that indicate neovascularization and calcification, including VEGF and BSP, were concurrently
\end{abstract}

Correspondence to: Dr Pin-Jing Hui, Department of Neurosurgery, The First Affiliated Hospital of Soochow University, 188 Shizi Street, Suzhou, Jiangsu 215006, P.R. China

E-mail: huipinjing1008@126.com

Professor Lan Xu, Department of Biochemical and Molecular of Medical College, Soochow University, 199 Renai Street, Suzhou, Jiangsu 215123, P.R. China

E-mail: xulan@suda.edu.cn

Key words: atherosclerosis, carotid plaque, matrix metalloproteinases, calcification factors, vulnerability elevated in vulnerable plaques. In addition, the protein levels of extracellular regulated kinase (ERK) and protein kinase C (PKC) were upregulated in vulnerable plaques. The current study provides novel insights into the MMP profiles of vulnerability plaques, and may assist in the development of novel methods for the diagnosis of plaque vulnerability and the prevention of plaque rupture.

\section{Introduction}

Carotid atherosclerotic plaque rupture and secondary thrombosis are two of the prominent causes of ischemic stroke (1). Carotid artery lesions account for $30 \%$ of ischemic strokes $(2,3)$. Plaques are divided into stable and vulnerable plaques (4). Vulnerable plaques refer to those that rupture easily, are prothrombotic and unstable (5). When a vulnerable plaque ruptures and becomes an embolus, the cerebral artery occludes, which eventually leads to ischemic stroke (6). As a result, it is of great clinical interest to stabilize vulnerable plaques.

Usually, plaques prone to rupture are morphologically characterized as having a thin fibrous cap overlaying a large lipid core (7). A fibrous cap primarily consists of vascular smooth muscle cells (VSMCs) and the extracellular matrix (ECM) (8). The remodeling and transformation of the ECM are important steps in the genesis and development of atherosclerosis (9). Under normal circumstances, a dynamic equilibrium exists between the generation and degradation of the ECM (10). Increasing evidence suggests that matrix metalloproteinases (MMPs) are one of the main factors degrading the ECM, which causes the rupture of plaques $(11,12)$. MMPs belong to the calcium-dependent zinc-containing protease superfamily (13). At present, $>20$ members of the MMP family have been identified (14). However, the roles that different MMPs serve in plaque stability have not been extensively studied. For example, Graham et al (15) revealed that the higher the serum MMP-9 level, the more unstable the plaque, suggesting that MMP-9 is associated with the plaque stability. Heo et al (16) revealed that MMP-2 and MMP-9 were highly expressed in the ulcerated plaques, suggesting that MMP-2 and MMP-9 may correlate with plaque rupture. In addition, only a small amount of data exists that allows for the quantitative and systematic 
evaluation of the expression of various types of MMPs in human carotid plaques, and determines the key factors that are associated with plaque vulnerability (17).

The vulnerability of plaques is a rather complicated issue in which neovascularization and calcification factors are involved $(18,19)$. Calcification is a pivotal event in the development of atherosclerosis $(20,21)$. During vascular calcification, VSMCs synthesize several osteogenic factors, including osteopontin (OPN) and bone sialoprotein 2 (BSP) $(22,23)$. OPN and BSP are considered to be important enzymes for the generation of calcium salt (24). However, the correlation between the associated factors, including BSP and OPN, and plaque stability remains unclear.

In the present study, carotid atherosclerotic plaques samples were obtained from patients undergoing carotid endarterectomies (CEA). Carotid atherosclerotic plaques were divided into stable and vulnerable groups. The expression levels of various types of MMPs, vascular endothelial growth factor (VEGF), BSP and OPN were compared between the two groups. The current study provided insight into the development of an improved method of plaque vulnerability diagnosis, and a possible therapy aimed at inhibiting plaque formation and stabilizing plaques to prevent plaque rupture.

\section{Materials and methods}

Carotid plaque specimens. Carotid plaques were collected from patients undergoing CEA in the Department of Neurosurgery of the First Affiliated Hospital of Soochow University (Suzhou, China). A total of 64 patients (56 men and 8 women, age 54-82 years) were recruited between February 2014 and February 2016. All patients underwent carotid duplex ultrasound, magnetic resonance imaging, computer tomography perfusion (CTP), computer tomography angiography (CTA) and/or digital subtraction angiography (DSA) prior to and following surgery. Plaques were defined as vulnerable if they had any one of the following characteristics: i) Active inflammation (monocyte/macrophage infiltration, occasionally with $\mathrm{T}$ lymphocyte infiltration); ii) a thin fibrous cap (thickness $<65 \mathrm{um}$ ), accompanied by large lipid core (lipid composition $>40 \%$ ); iii) endothelial cell loss with platelet aggregation on the surface of plaques; iv) rupture of the fibrous cap; v) severe vessel stenosis $>90 \%$; or if they had a minimum of two of the following characteristics: i) Calcified nodules in plaque surface; ii) intraplaque hemorrhage; iii) dysfunction of endothelial cells in the plaque; iv) vascular remodeling (25). Based on the aforementioned criteria, the atherosclerotic carotid plaque samples were divided into 30 stable plaques and 34 vulnerable plaques. Written consent was obtained from each patient. The experimental protocol was approved by the Ethics Committee of the First Affiliated Hospital of Soochow University (approval no. 2011310044).

$R N A$ isolation and reverse transcription-quantitative polymerase chain reaction (RT-qPCR). The plaque segments were homogenized in liquid nitrogen. Total RNA was extracted from the homogenates using TRIzol reagent (Invitrogen; Thermo Fisher Scientific, Inc., Waltham, MA, USA). RNA concentration was quantified using a NanoDrop ${ }^{\mathrm{TM}} 2000$ Spectrophotometer (Thermo Fisher Scientific, Inc.). cDNA was synthesized using the ReverTra Ace ${ }^{\circledR}$ qPCR RT kit (Toyobo Life Science, Osaka, Japan) according to the manufacturer's protocol. Primers were synthesized by and purchased from Sangon Biotech Co., Ltd. (Shanghai, China) (Table I). qPCR analysis was performed using SYBR ${ }^{\circledR}$ Green Realtime PCR Master mix (Toyobo Life Science) and an ABI PRISM 7000 sequence detection system (Applied Biosystems; Thermo Fisher Scientific, Inc.). The thermocycling conditions were as follows: $95^{\circ} \mathrm{C}$ for $60 \mathrm{sec}, 40$ cycles of $95^{\circ} \mathrm{C}$ for $15 \mathrm{sec}$ and $60^{\circ} \mathrm{C}$ for $60 \mathrm{sec}$. Relative mRNA expression was quantified using the $2^{-\Delta \Delta \mathrm{Cq}}$ method (26). GAPDH served as an internal reference gene. All RT-qPCR analyses were repeated three times.

Protein extraction and western blotting. The plaque segments were homogenized in liquid nitrogen and lysed with mammalian protein extraction reagent supplemented with $1 \%$ protease inhibitors (both Beijing ComWin Biotech, Co., Ltd., Beijing, China) at $4^{\circ} \mathrm{C}$ for $30 \mathrm{~min}$. Protein concentration was determined by a BCA assay using the bicinchonininc acid protein assay kit (Pierce; Thermo Fisher Scientific, Inc.). Protein samples $(10 \mu \mathrm{g})$ were mixed with an SDS-PAGE protein loading buffer (Beyotime Institute of Biotechnology, Haimen, China) and boiled for $10 \mathrm{~min}$. Proteins were separated on a $12 \%$ SDS-PAGE gel and transferred to nitrocellulose (NC) membranes (EMD Millipore, Billerica, MA, USA). The NC membranes were then blocked with $5 \%$ fat free milk in Tris-buffered saline with $0.5 \%$ Tween-20 (TBST) for $1 \mathrm{~h}$ at room temperature. The membranes were incubated with primary antibodies at $4{ }^{\circ} \mathrm{C}$ overnight. The primary antibodies used in the current study were as follows: Mouse anti-MMP2 (1:1,000; cat. no. Ab80737), rabbit anti-MMP14 (1:1,000; cat. no. Ab51074), mouse anti-MMP25 (1:1,000; cat. no. GR26383-3), rabbit anti-OPN (1:1,000; cat. no. Ab104302) (all Abcam, Cambridge UK), mouse anti-VEGF (1:1,000; cat. no. 500-M88; PeproTech, Rocky Hill, NJ, USA), goat anti-BSP (1:1,000; cat. no AF4014; R\&D Systems, Inc., Minneapolis, MN, USA), rabbit anti-extracellular regulated kinase (ERK; 1:1,000; cat. no. YT1624) and rabbit anti-protein kinase C (PKC; 1:1,000; cat. no. YT3752) (both ImmunoWay Biotechnology Company, Plano, TX, USA) and mouse anti-GAPDH (1:1,000; cat. no. AG019-1; Beyotime Institute of Biotechnology). Then, the NC membrane was washed twice with TBST. The membranes were then incubated with a horseradish peroxidase-conjugated anti-rabbit secondary antibodies (1:1,000; cat. no. SA00001-2; ProteinTech Group, Inc., Chicago, IL, USA), anti-mouse secondary antibodies (1:1,000; A0216) and anti-goat secondary antibodies (1:1,000; A0181) (both Beyotime Institute of Biotechnology) for $2 \mathrm{~h}$ at room temperature. The protein bands were developed using an enhanced chemiluminescence kit (Beijing ComWin Biotech, Co., Ltd.). GAPDH was used as the internal control. The protein brands were scanned and quantified using ImageJ software (v1.8.0; National Institutes of Health, Bethesda, MD, USA).

Histology and immunohistochemistry. Paraffin sections were subjected to immunohistochemical staining for MMP-2, -14 and -25 . The samples were fixed in $10 \%$ formalin and embedded into paraffin at room temperature until they set. Then, the paraffin section was cut into $4-\mu$ m-thick slices. The sections were incubated at $65^{\circ} \mathrm{C}$ overnight. Sections were de-paraffinized with xylene for $5 \mathrm{~min}$ and re-hydrated 
Table I. Sequences of primers used for tissue sample of human.

Primer sequence (5'-3')

\begin{tabular}{lll}
\cline { 2 - 2 } Gene & \multicolumn{1}{c}{ Forward } & \multicolumn{1}{c}{ Reverse } \\
\hline MMP-1 & GAAGAATGATGGGAGGCAAG & CAGGGTTTCAGCATCTGGTT \\
MMP-2 & TATGGCTTCTGCCCTGAGAC & CACACCACATCTTTCCGTCA \\
MMP-3 & ATCCCGAAGTGGAGGAAAAC & AGCCTGGAGAATGTGAGTG \\
MMP-7 & ACCGTGCTGTGTGCTGTGT & GTCCTGAGCCTGTTCCCACT \\
MMP-9 & TCTTCCCCTTCACTTTCCTG & CCCACTTCTTGTCGCTGTC \\
MMP-12 & CATGAACCGTGAGGATGTTG & GGCAAAAACCACCAAAATG \\
MMP-13 & GCAGTCTTTCTTCGGCTTAGAG & GTATTCACCCACATCAGGAACC \\
MMP-14 & GCAGAAGTTTTACGGCTTGC & GCAGAAGTTTACGGCTTGC \\
MMP-15 & TTATGGCTACCTGCCTCAGC & TCCACTCCTTGGTCTCTTCG \\
MMP-16 & TGATTTACAGGGCATCCAGA & TCATTTTTCCTTGGGTCAGC \\
MMP-17 & TGACCACACGAGGCACAT & CCATCCAGCACTTTCCAGTA \\
MMP-24 & TGGAGGCAAAAACACATCAC & TCAAAGGTCAGTGGGGTCA \\
MMP-25 & GCAGCAACTCTATGGGAAGG & ATCAGGGATGGGGAAGGAT \\
GAPDH & AATCCCATCACCATCTTCCA & AAATGAGCCCCAGCCTTCT \\
\hline
\end{tabular}

MMP, matrix metalloproteinase.

with an alcohol gradient (100, 90 and 70\%) for 3 min each time. Following washing with TBS 3 times, antigen retrieval was performed and the sections were soaked in $3 \% \mathrm{H}_{2} \mathrm{O}_{2}$ for $10 \mathrm{~min}$ at room temperature. Then, after washing three times the antigen sites were exposed using a microwave oven with $0.05 \%$ phosphate buffer. The sections were incubated with FBS at $37^{\circ} \mathrm{C}$ for 30 mins. After washing with PBS 3 times, the sections were incubated with rabbit and mouse monoclonal antibodies overnight at $4^{\circ} \mathrm{C}$. PBS $0.01 \mathrm{M}$ was used to was the tissue sections three times. The sections were then incubated with horseradish peroxidase-conjugated mouse anti-rabbit secondary antibodies (1:500; cat. no. AB11053; BBI Solutions, Cardiff, UK) at $37^{\circ} \mathrm{C}$ for $30 \mathrm{~min}$. The primary antibodies used in the current study were as follows: Mouse anti-MMP2 (1:500; cat. no. ab80737), rabbit anti-MMP14 (1:500; cat. no. ab51074), mouse anti-MMP25 (1:500; cat. no. GR26383-3) (all Abcam). After washing the following chromogenic agents were added: $0.05 \% \mathrm{DAB}, 5 \% \mathrm{H}_{2} \mathrm{O}_{2}$ and $0.05 \%$ phosphate buffer. The sections were washed, counterstained with hematoxylin for $30 \mathrm{sec}$ at room temperature, dehydrated and protected with coverslips. ImageJ software was used for density analysis of immunohistochemical results. The results were observed and captured at magnification, x200 under an electron microscope. The gradational distinction was based on positivity cell numbers and staining intensity. The indicators,+++ and +++ were used to indicate the strength of the stain as follows: + , light staining of plaques as bright yellow; ++ , medium staining of plaques as pale brown; +++ , dark staining of plaques as dark brown. Plaque and tissue sections were also stained with hematoxylin and eosin (H\&E) for 2-3 min at room temperature, for histologic observation.

Statistical analysis. All statistical analyses were performed with SPSS 20.0 software (IBM Corp, Armonk, NY, USA).
Data were analyzed by paired t-tests or, for immunohistochemical results, Chi-squared tests. Values are presented as mean \pm standard deviation. $\mathrm{P}<0.05$ was considered to indicate a statistically significant difference.

\section{Results}

Patient characteristics and clinical data. As determined by ultrasonography and $H \& E$ staining, all the carotid plaques were divided into 30 stable plaques and 34 vulnerable plaques. As presented in Fig. 1A, stable plaques exhibited uniform echoes and smooth surfaces. Conversely, vulnerable plaques exhibited uneven echoes and irregular shapes, and color Doppler blood signals were occasionally exhibited (Fig. 1B). The H\&E staining of the stable plaques revealed the smooth and thick fibrous cap (Fig. 1C). In contrast, the majority of vulnerable plaques contained discontinuous and thin fibrous caps with thrombi, neovascularization and lipid cores (Fig. 1D). Table I presents the demographics, risk factors for ischemic stroke, degrees of stenosis, diseases and clinical symptoms of the patients involved in the present study. As demonstrated in Table I, the development of vulnerable plaques was associated with sex. Males were significantly more likely to develop vulnerable carotid plaques compared with females. In addition, patients with vulnerable plaques had a statistically higher number of transient ischemic attacks (TIA; Table II). No significant differences were identified in the other symptoms and stroke risk factors between the two groups.

mRNA levels of MMP-2, -7, -9 and -14 are upregulated in vulnerable plaques. MMPs are metalloproteases important in the destabilization of plaques (27). To understand which metalloproteases are determinants for the onset of vulnerable plaques, the mRNA levels of various MMPs were analyzed 

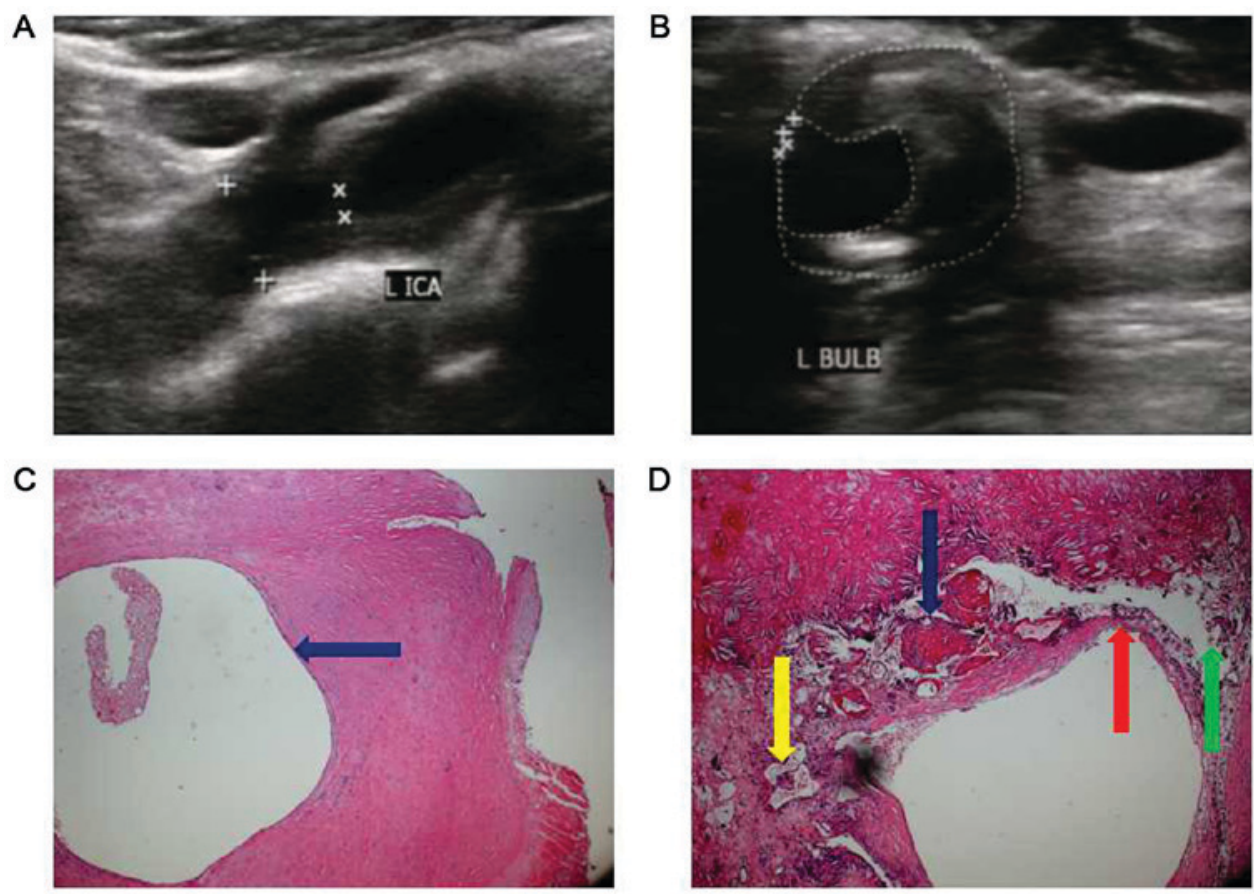

Figure 1. Characteristics of stable and vulnerable plaques. Features of (A) stable and (B) vulnerable plaques by ultrasound. The white crosses represent a measure of the diameter and area-narrowing rate of blood vessels caused by the plaque. The dotted line is an area measurement that represents the narrowing of blood vessels caused by the plaque. (C) Characteristics of a stable plaque by H\&E staining. The blue arrow indicated a smooth and thick fibrous cap. (D) Characteristics of vulnerable plaque by H\&E staining. Arrows indicate a discontinuous and thin fibrous cap (red), a thrombus (blue), neovascularization (yellow) and a lipid core (green) in the vulnerable plaque. Magnification, x10. H\&E, hematoxylin and eosin; LICA, left internal carotid artery; L BULB, left carotid bulb.

in the plaque segments. The mRNA level of MMP-1, $-2,-3$, $-7,-9,-12,-13,-14,-15,-16,-17,-24$ and -25 were compared between the stable and vulnerable plaque segments (Fig. 2). The expression levels of MMP-2, -9, -14 were significantly higher ( $>20$-fold) in the vulnerable plaques compared with the stable plaques; MMP-7 expression levels were also significantly raised ( $>2$-fold) in vulnerable plaques compared with stable plaques. No significant differences were identified in the mRNA levels of other MMPs between the two plaque types.

MMP-2 and MMP-14 protein levels are increased in homogenized vulnerable plaques. MMP-7 and MMP-9 have been extensively studied prior to the current study (28-30), therefore MMP-2 and MMP-14 were selected for quantification by western blotting; MMP-2 and MMP-14 also demonstrated the greatest expression fold change in Fig. 2. MMP-25, whose expression was not altered between the two types of plaques, was selected as a negative control. Consistent with the gene expression, the protein levels of MMP-2 and MMP-14 were significantly upregulated ( $>2$-fold) in vulnerable plaques compared with those in stable plaques (Fig. 3A). Consistent with the mRNA level, no significant difference was identified in the protein levels of MMP-25 between the two groups.

Angiogenesis and calcification factors are altered in vulnerable plaques. To further investigate the biochemical characteristics of different types of plaques, the protein levels of vascularization marker VEGF and calcification markers BSP and OPN were analyzed. VEGF expression was significantly upregulated in vulnerable plaques compared with the stable group (Fig. 3A). The protein expression of BSP was significantly increased while OPN protein levels were significantly decreased in vulnerable plaques compared with stable plaques (Fig. 3B).

To gain more insight into the signaling pathway that is activated and may mediate the pathogenesis of plaque vulnerability, the steady state protein level of key mediators of various signaling pathways was also analyzed. No significant differences were identified in proto-oncogene c-Fos, RAC-alpha serine/threonine-protein kinase, runt-related transcription factor 2, protein Wnt-16, mothers against decapentaplegic homolog (SMAD)1, SMAD2,3 proteins between the stable and vulnerable groups of carotid plaques (data not shown). However, the steady state protein levels of ERK and PKC were significantly increased in vulnerable plaques compared with stable plaques (Fig. 3B).

Protein levels of MMP-2 and MMP-14 are increased in vulnerable plaque tissue sections. The protein levels of MMP-2 and MMP-14 were upregulated in vulnerable plaques compared with stable plaques. To localize MMP-2 and MMP-14 expression within the two groups of carotid plaque, serial tissue sections were subjected to immunostaining (Fig. 4). $\mathrm{H} \& \mathrm{E}$ staining revealed that macrophages and foam cells were primarily distributed at the location of the plaque rupture and were more widely distributed in the vulnerable plaque group than in the stable plaque group. The two groups of carotid plaques were positively stained with MMP-2 and MMP-14 at different intensities. Staining for MMP-2 and MMP-14 was markedly more intense in vulnerable plaques compared with stable plaques. MMP-2 and MMP-14 staining was positive in the atherosclerotic lesions, and markedly increased in the 
Table II. Clinical index of the 64 patients recruited in this study.

\begin{tabular}{lccc}
\hline Characteristic & $\begin{array}{c}\text { Stable } \\
(\mathrm{n}=30)\end{array}$ & $\begin{array}{c}\text { Vulnerable } \\
(\mathrm{n}=34)\end{array}$ & P-value \\
\hline Age mean (years) & 67 & 69 & $\mathrm{NS}$ \\
Sex (male/female) & $23 / 7$ & $33 / 1$ & $<0.05^{\mathrm{a}}$ \\
Amaurosis fugax & $3(10 \%)$ & $5(15 \%)$ & $\mathrm{NS}$ \\
Transient ischemic attack & $5(17 \%)$ & $13(38 \%)$ & $<0.05$ \\
Stroke & $4(13 \%)$ & $7(20 \%)$ & $\mathrm{NS}$ \\
Hypertension & $6(20 \%)$ & $9(26 \%)$ & $\mathrm{NS}$ \\
Diabetes & $3(6 \%)$ & $4(12 \%)$ & $\mathrm{NS}$ \\
Hyperlipidemia & $7(23 \%)$ & $8(24 \%)$ & $\mathrm{NS}$ \\
Smoking habit & $12(40 \%)$ & $15(44 \%)$ & $\mathrm{NS}$ \\
Statins & $16(53 \%)$ & $14(44 \%)$ & $\mathrm{NS}$ \\
Degree of stenosis & $4 / 26$ & $7 / 27$ & $\mathrm{NS}$ \\
(medium/slight) & & & \\
\hline
\end{tabular}

${ }^{a}$ As determined using a Chi-square test. The diagnostic criteria for different clinical index are as follows: Hypertension (systolic pressure, $\geq 160 \mathrm{mmHg}$ ), hyperlipidemia (total cholesterol, $\geq 220 \mathrm{mg} / \mathrm{dl}$ ), diabetes (fasting blood glucose, $\geq 126 \mathrm{mg} / \mathrm{dl}$ ) and a history of continuous smoking ( $\geq 15$ year). NS, not significant.

breakdown region of the fibrous cap. Consistent with the protein level, density analysis revealed that the immunohistochemical staining of MMP-2 (Table III) and MMP-14 (Table IV) was significantly more intense in samples from vulnerable plaques compared with stable plaques. It was observed that staining for MMP-25 was not significantly different between the vulnerable and stable plaques (data not shown).

\section{Discussion}

Atherosclerosis is the critical risk factor associated with the onset of stroke. Carotid stenosis is a serious long-term outcome of atherosclerosis $(31,32)$. Carotid atherosclerotic plaques, particularly vulnerable carotid plaques were closely associated with ischemic stroke (33). In the current study, 64 patients were recruited and carotid plaques were collected following CEA. The collected carotid plaques were divided into the stable and vulnerable groups. Patients with vulnerable plaques had significantly higher numbers of TIA. According to previous studies, these patients exhibit a higher risk of acute cerebrovascular events caused by the rupture of vulnerable plaques (34-36).

An increasing number of studies have suggested that MMPs are the foremost factors that degrade the ECM and cause the rupture of vulnerable plaques $(37,38)$. MMPs are grouped into five types based on their structure and substrate specificity; these include collagenases (MMP-1, -8,-13 and -18), gelatinases (MMP-2 and -9), stromelysins (MMP-3, -7, -10, -11 and -12), membrane-type MMPs (MMP-14, -15,-16,-17,-24 and -25) and the other MMPs (39). In the current study, the expression levels of various MMPs in human carotid plaques were investigated; it was demonstrated that the expression levels of MMP-2, -7, -9 and -14 were elevated in vulnerable plaques, particularly

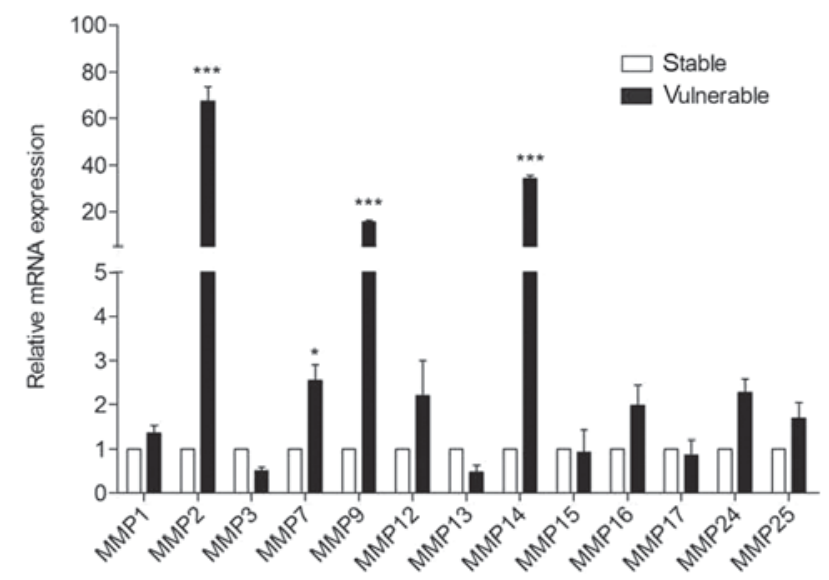

Figure 2. mRNA level of MMP-2, -7, -9 and -14 are upregulated in vulnerable plaques. The mRNA expression levels of MMPs were detected by reverse transcription-quantitative polymerase chain reaction and quantified using the $2^{-\triangle \Delta C q}$ method. Data are presented as the mean \pm standard deviation. ${ }^{*} \mathrm{P}<0.05$, ${ }^{* * * *} \mathrm{P}<0.001$ vs. the stable plaques group. MMP, matrix metalloproteinase.

MMP-2 and -14. Additionally, immunohistochemistry demonstrated that MMP-2 and -14 were predominantly distributed in the ruptured region of vulnerable plaques, which was the same area where VSMCs and macrophage aggregated (40). Studies have confirmed that the overexpression of MMP-2 and -14 serves a critical role in the cell migration and growth of endothelial cells, and the formation of new blood vessels by adjusting the ECM environment $(41,42)$.

The activation of MMP-2 was associated with the increased expression of MMP-14 (41). MMP-14 not only acts as a protease, but also a signaling molecule, which affects the pathological and physiological functions of cells (42). As is well known, the ECM is important for remodeling the fibrous cap (43). Vulnerable plaques are morphologically characterized as possessing a thin fibrous cap (44). In the present study, elevated expression of MMP-2 and -14 was observed, which may lead to ECM degradation, resulting in further thinning of the shoulders of the fibrous cap and the rupture of plaques. Previous studies have suggested that the expression of MMP-25 is low in atherosclerosis $(45,46)$. Notably, no significant difference in MMP-25 protein level was identified between stable and vulnerable plaques in the present study, suggesting that MMP-25 did not contribute to the plaque vulnerability and may have no role in the rupture of vulnerable plaques. The data from the current study indicated that MMP-2 and -14 may be valuable clinical biomarkers for plaque vulnerability.

VEGF serves a decisive role in promoting instability by inducing angiogenesis in plaques (47). The current study verified that the expression of VEGF was significantly higher in vulnerable plaques. Angiogenesis is a critical factor in the process of atherosclerosis, which can induce intraplaque hemorrhage and rupture. Plaque calcification gradually forms during atherosclerosis (48). Various studies suggested that calcification may alter the stability of atherosclerotic plaques $(24,49)$. During calcification, VSMCs synthesize several osteogenic factors, including BSP (50). Fedarko et al (51) revealed that BSP specifically binds proMMP-2 and active MMP-2, while OPN binds proMMP-3 and active MMP-3. It has been 

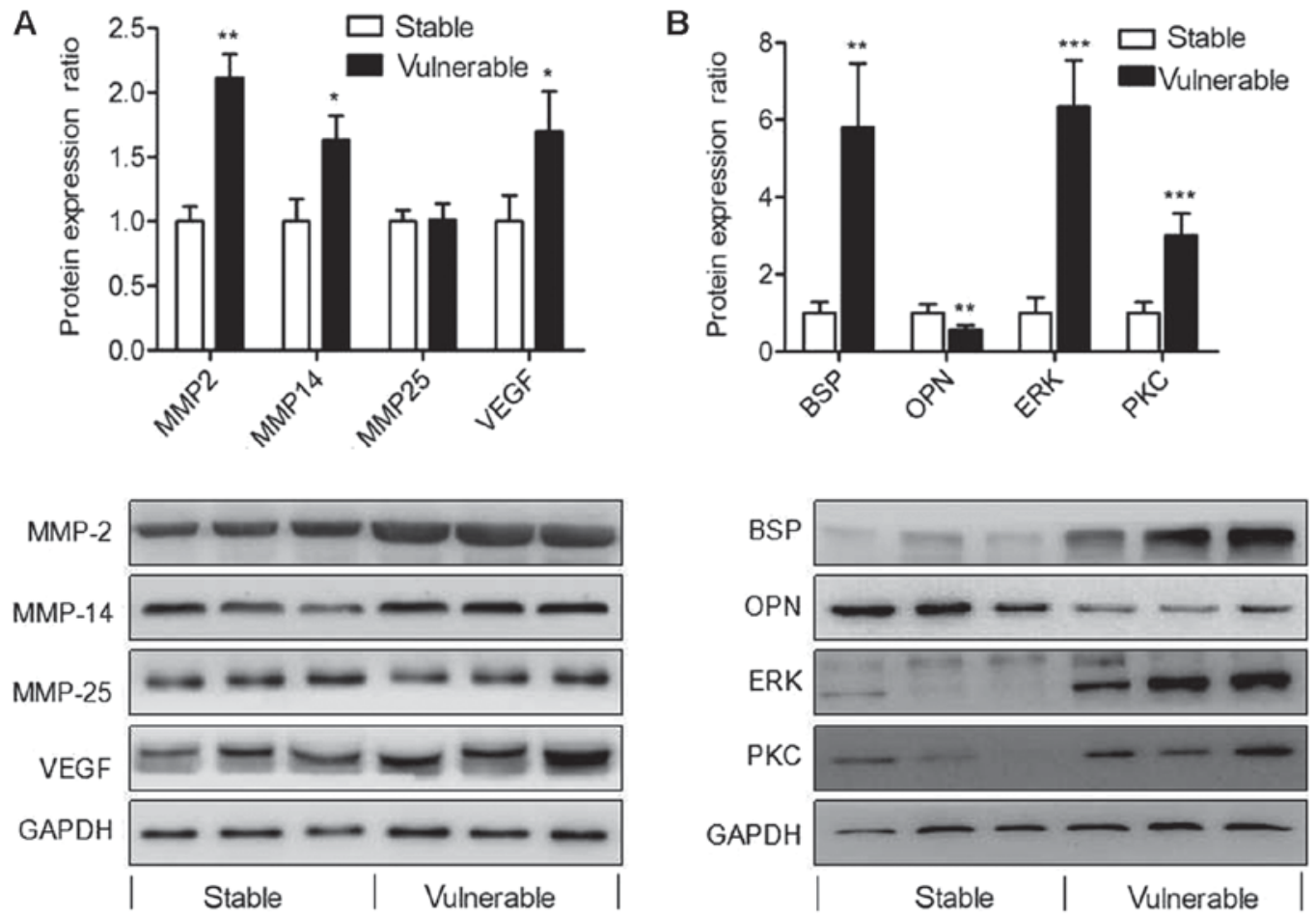

Figure 3. Protein level of MMPs, associated factors and signaling pathway proteins altered in vulnerable plaques. Western blot analyses and quantification of (A) MMP-2, MMP-14, MMP-25, VEGF, (B) BSP, OPN, ERK and PKC protein levels in stable and vulnerable plaques. Data are presented the mean \pm standard deviation. ${ }^{*} \mathrm{P}<0.05,{ }^{* *} \mathrm{P}<0.01$ and ${ }^{* * *} \mathrm{P}<0.001$ vs. the stable plaques group. MMP, matrix metalloproteinase; VEGF, vascular endothelial growth factor; BSP, bone sialoprotein 2; OPN, osteopontin; ERK, mitogen-activated protein kinase 3; PKC, protein kinase C.

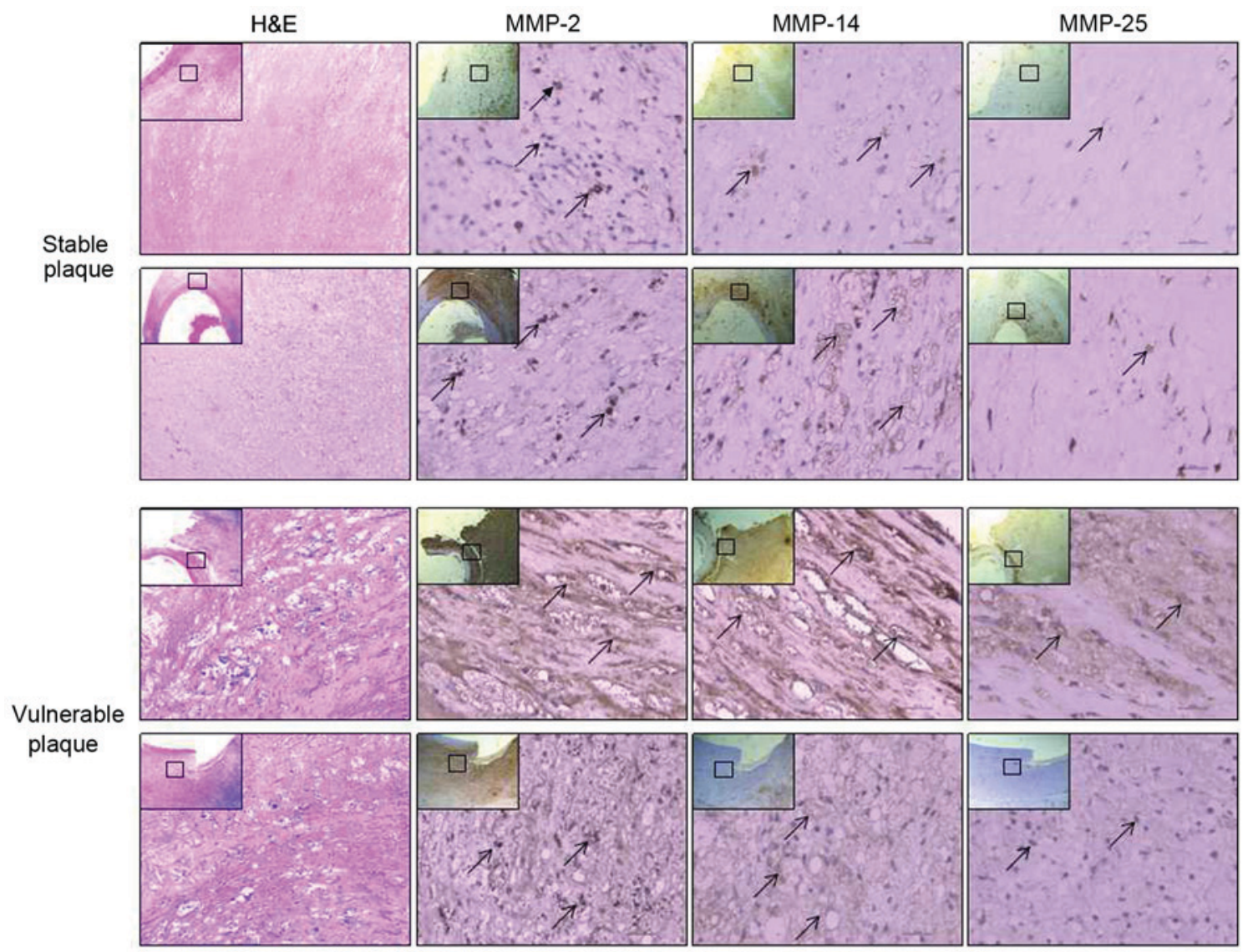

Figure 4. Protein level of MMP-2 and MMP-7 are increased in vulnerable plaque tissue sections. H\&E staining and immunohistochemical analysis of MMP-2, -14 and -25 in stable and vulnerable carotid plaques. The insert panels are at a lower magnification (magnification, $\mathrm{x} 4$ ) than the larger panels (magnification, $\mathrm{x} 40$ ). The black arrows indicate positive staining. Bright yellow, light brown and dark brown staining indicates light, medium and severe staining, respectively. MMP, matrix metalloproteinase; H\&E, hematoxylin and eosin. 
Table III. Statistics of immunohistochemical matrix metalloproteinase 2 staining in stable and vulnerable plaques.

\begin{tabular}{lrrrr}
\hline Group & + & ++ & +++ & Total \\
\hline Stable & 16 & 10 & 4 & 30 \\
Vulnerable $^{\mathrm{a}}$ & 3 & 12 & 19 & 34 \\
Total & 19 & 22 & 23 & 64 \\
\hline
\end{tabular}

,+++ and +++ indicate light, medium and severe staining, respectively. + , light staining of plaques as bright yellow; ++ , medium staining of plaques as pale brown; +++, dark staining of plaques as dark brown. ${ }^{\mathrm{a}} \mathrm{P}<0.001 \mathrm{vs}$. the group of stable plaques. These results were analyzed by a Chi-square test.

Table IV. Statistics of immunohistochemical matrix metalloproteinase 14 staining in stable and vulnerable plaques.

\begin{tabular}{lrrrr}
\hline Group & + & ++ & +++ & Total \\
\hline Stable & 17 & 11 & 2 & 30 \\
Vulnerable $^{\mathrm{a}}$ & 5 & 13 & 16 & 34 \\
Total & 22 & 24 & 18 & 64 \\
\hline
\end{tabular}

,+++ and +++ indicate light, medium and severe staining, respectively. ${ }^{a} \mathrm{P}<0.001$ vs. the group of stable plaques. These results were analyzed by a Chi-square test.

demonstrated that BSP exhibits hydroxyapatite nucleation activity and may serve important roles in the initiation of and calcification during atherosclerosis $(52,53)$. OPN is a potent inhibitor of mineralization (54). OPN prevents ectopic calcium deposition and is a strong, inducible inhibitor of vascular calcification (55). The current study revealed that the overexpression of BSP and attenuation of OPN may be associated with plaque vulnerability.

A number of studies investigating the association between MMPs and vascular biology have demonstrated that a number of factors leading to angiogenesis and vascular remodeling-associated diseases regulate MMP expression and activation, including hemodynamics, oxidative stress, inflammation, hormonal factors and hypoxia $(56,57)$. Studies have revealed that low fluid shear stress-induced MMP expression involves the integrin, p38 mitogen-activated protein kinase 1 or ERK1/2-nuclear factor (NF)- $\kappa \mathrm{B}$ signaling pathways $(58,59)$. Studies have indicated that OPN may promote the expression of MMP-2 through the ERK signaling pathways $(56,60)$. In the present study, various signaling pathways were investigated by western blotting and it was demonstrated that ERK and PKC were significantly higher in vulnerable plaques, indicating that they may be involved the process of plaque rupture. It was hypothesized that activation of ERK and PKC may also increase MMP-2 and -14 expression. Simultaneously, ERK and PKC were activated to promote BSP secretion and decrease OPN expression, which promotes the upregulation of MMP-2 and -14 , and increase the likelihood of shoulder rupture of atherosclerotic plaques.
In summary, the current study demonstrated that MMP-2 and -14 were elevated in vulnerable plaques and may serve a significant role in rupture of carotid plaques. The differential regulation of factors, including VEGF and BSP, were also associated with the plaque vulnerability. More studies are required to demonstrate the association of the aforementioned factors with plaque rupture events and the accurate prediction of vulnerable plaques. Mechanistic studies investigating how plaque rupture is regulated should be performed for the development of possible therapies that stabilize vulnerable plaques and reduce the occurrence of ischemic stroke.

\section{Acknowledgements}

The authors would like to thank Professor Lan Xu (Soochow University, Suzhou, China) for her assistance and advice.

\section{Funding}

This work was supported by the National Natural Science Foundation of China (grant no. 31401173) and the Natural Science Foundation of Jiangsu Province (grant no. BK20140329). This work was also supported by the People's Livelihood Technology Demonstration Project of Suzhou (grant no. SS201714) and the Province Care Health Care Research Project of Jiangsu (grant no. BJ17010).

\section{Availability of data and materials}

The datasets used and/or analyzed during the current study are available from the corresponding author on reasonable request.

\section{Authors' contributions}

ZYG contributed to the acquisition and analysis of the data, performed the basic experiments and was involved in the drafting of the manuscript. $\mathrm{PJH}$ contributed to the conception and design of the study and gave final approval of the version to be published. LX contributed to the conception and design of the data analysis, assisted with the experiments and was involved in revising the manuscript critically for intellectual content. BZ and YHY assessed the properties of the plaques using vascular ultrasound and assisted with the collection of specimens. SSG and JJL assisted with the experiments. All authors read and approved the final version of the manuscript.

\section{Ethics approval and consent to participate}

Written consent was obtained from each patient. The experimental protocol was approved by the ethics committee of the First Affiliated Hospital of Soochow University (approval no. 2011310044).

\section{Patient consent for publication}

The patients have provided written informed consent for the publication of any associated data and accompanying images. 


\section{Competing interests}

The authors declare that they have no competing interests.

\section{References}

1. Liu XS, Zhao HL, Cao Y, Lu Q and Xu JR: Comparison of carotid atherosclerotic plaque characteristics by high-resolution black-blood MR imaging between patients with first-time and recurrent acute ischemic stroke. AJNR Am J Neuroradiol 33: $1257-1261,2012$.

2. Rosamond W, Flegal K, Friday G, Furie K, Go A, Greenlund K, Haase N, Ho M, Howard V, Kissela B, et al: Heart disease and stroke statistics-2007 update: A report from the American Heart Association Statistics Committee and Stroke Statistics Subcommittee. Circulation 115: e69-e171, 2007.

3. Kuk M, Wannarong T, Beletsky V, Parraga G, Fenster A and Spence JD: Volume of carotid artery ulceration as a predictor of cardiovascular events. Stroke 45: 1437-1441, 2014.

4. Jin G: The relationship between serum CXCL16 level and carotid vulnerable plaque in patients with ischemic stroke. Eur Rev Med Pharmacol Sci 21: 3911-3915, 2017.

5. Chatzizisis YS, Antoniadis AP, Wentzel JJ and Giannoglou GD: Vulnerable plaque: The biomechanics of matter. Atherosclerosis 236: 351-352, 2014.

6. Badimon L and Vilahur G: Thrombosis formation on atherosclerotic lesions and plaque rupture. J Intern Med 276: 618-632, 2014.

7. Yonetsu T and Jang IK: Advances in Intravascular Imaging: New insights into the vulnerable plaque from imaging studies. Korean Circ J 48: 1-15, 2018.

8. Virmani R, Burke AP, Farb A and Kolodgie FD: Pathology of the vulnerable plaque. J Am Coll Cardiol 47 (8 Suppl): C13-C18, 2006.

9. Souilhol C, Harmsen MC, Evans PC and Krenning G: Endothelial-mesenchymal transition in atherosclerosis. Cardiovasc Res 114: 565-577, 2018.

10. Heeneman S, Cleutjens JP, Faber BC, Creemers EE, van Suylen RJ, Lutgens E, Cleutjens KB and Daemen MJ: The dynamic extracellular matrix: Intervention strategies during heart failure and atherosclerosis. J Pathol 200: 516-525, 2003.

11. Galis ZS, Sukhova GK, Lark MW and Libby P: Increased expression of matrix metalloproteinases and matrix degrading activity in vulnerable regions of human atherosclerotic plaques. J Clin Invest 94: 2493-2503, 1994.

12. Jones CB, Sane DC and Herrington DM: Matrix metalloproteinases: A review of their structure and role in acute coronary syndrome. Cardiovasc Res 59: 812-823, 2003.

13. Singh T, Adekoya OA and Jayaram B: Understanding the binding of inhibitors of matrix metalloproteinases by molecular docking, quantum mechanical calculations, molecular dynamics simulations, and a MMGBSA/MMBappl study. Mol Biosyst 11: 1041-1051, 2015

14. Nagase H and Woessner JF Jr: Matrix metalloproteinases. J Biol Chem 274: 21491-21494, 1999.

15. Graham CA, Chan RW, Chan DY, Chan CP, Wong LK and Rainer TH: Matrix metalloproteinase 9 mRNA: An early prognostic marker for patients with acute stroke. Clin Biochem 45: 352-355, 2012

16. Heo W, Lee YS, Son CH, Yang K, Park YS and Bae J: Radiation-induced matrix metalloproteinases limit natural killer cell-mediated anticancer immunity in NCI-H23 lung cancer cells. Mol Med Rep 11: 1800-1806, 2015.

17. Han Y, Mao X, Wang L, Liu J, Wang D, Cheng H and Miao G: increased levels of soluble cluster of differentiation 40 ligand, matrix metalloproteinase 9, and matrix metalloproteinase 2 are associated with carotid plaque vulnerability in patients with ischemic cerebrovascular disease. World Neurosurg 105: 709-713, 2017

18. Virmani R, Kolodgie FD, Burke AP, Finn AV, Gold HK, Tulenko TN, Wrenn SP and Narula J: Atherosclerotic plaque progression and vulnerability to rupture: Angiogenesis as a source of intraplaque hemorrhage. Arterioscler Thromb Vasc Biol 25: 2054-2061, 2005.

19. Foley CJ, Fanjul-Fernández M, Bohm A, Nguyen N, Agarwal A, Austin K, Koukos G, Covic L, López-Otín C and Kuliopulos A: Matrix metalloprotease 1a deficiency suppresses tumor growth and angiogenesis. Oncogene 33: 2264-2272, 2014.
20. Doherty TM, Asotra K, Fitzpatrick LA, Qiao JH, Wilkin DJ, Detrano RC, Dunstan CR, Shah PK and Rajavashisth TB: Calcification in atherosclerosis: Bone biology and chronic inflammation at the arterial crossroads. Proc Natl Acad Sci USA 100: 11201-11206, 2003.

21. Wahlgren CM, Zheng W, Shaalan W, Tang J and Bassiouny HS: Human carotid plaque calcification and vulnerability. Relationship between degree of plaque calcification, fibrous cap inflammatory gene expression and symptomatology. Cerebrovasc Dis 27: 193-200, 2009.

22. Charo IF and Ransohoff RM: The many roles of chemokines and chemokine receptors in inflammation. N Engl J Med 354: 610-621, 2006

23. Charo IF and Taubman MB: Chemokines in the pathogenesis of vascular disease. Circ Res 95: 858-866, 2004.

24. Kruger TE, Miller AH, Godwin AK and Wang J: Bone sialoprotein and osteopontin in bone metastasis of osteotropic cancers. Crit Rev Oncol Hematol 89: 330-341, 2014.

25. Naghavi M, Libby P, Falk E, Casscells SW, Litovsky S, Rumberger J, Badimon JJ, Stefanadis C, Moreno P, Pasterkamp G, et al: From vulnerable plaque to vulnerable patient: A call for new definitions and risk assessment strategies: Part I. Circulation 108: 1664-1672, 2003.

26. Livak KJ and Schmittgen TD: Analysis of relative gene expression data using real-time quantitative PCR and the 2(-Delta Delta C(T)) method. Methods 25: 402-408, 2001.

27. Müller A, Krämer SD, Meletta R, Beck K, Selivanova SV, Rancic Z, Kaufmann PA, Vos B, Meding J, Stellfeld T, et al: Gene expression levels of matrix metalloproteinases in human atherosclerotic plaques and evaluation of radiolabeled inhibitors as imaging agents for plaque vulnerability. Nucl Med Biol 41: $562-569,2014$.

28. Hakimzadeh N, Pinas VA, Molenaar G, de Waard V, Lutgens E, van Eck-Smit BLF, de Bruin K, Piek JJ, Eersels JLH, Booij J, et al: Novel molecular imaging ligands targeting matrix metalloproteinases 2 and 9 for imaging of unstable atherosclerotic plaques. PLoS One 12: $\mathrm{e} 0187767,2017$.

29. Razuvaev A, Ekstrand J, Folkersen L, Agardh H, Markus D, Swedenborg J, Hansson GK, Gabrielsen A, Paulsson-Berne G, Roy J and Hedin U: Correlations between clinical variables and gene-expression profiles in carotid plaque instability. Eur J Vasc Endovasc Surg 42: 722-730, 2011.

30. Ragino IuI, Cherniavskiǐ AM, Polonskaia IaV, Volkov AM, Semaeva EV, Tsymbal SIu and Voevoda MI: Changes in proinflammatory cytokine and destructive metalloproteinase levels during formation of unstable atherosclerotic plaque. Kardiologiia 49: 43-49, 2009 (In Russian).

31. Andrews JPM, Fayad ZA and Dweck MR: New methods to image unstable atherosclerotic plaques. Atherosclerosis 272: 118-128, 2018.

32. Lapenna D, Ciofani G, Pierdomenico SD, Giamberardino MA Ucchino $S$ and Davì G: Association of serum bilirubin with oxidant damage of human atherosclerotic plaques and the severity of atherosclerosis. Clin Exp Med 18: 119-124, 2018.

33. Pelisek J, Eckstein HH and Zernecke A: Pathophysiological mechanisms of carotid plaque vulnerability: Impact on ischemic stroke. Arch Immunol Ther Exp (Warsz) 60: 431-442, 2012.

34. Sakakura K, Nakano M, Otsuka F, Ladich E, Kolodgie FD and Virmani R: Pathophysiology of atherosclerosis plaque progression. Heart Lung Circ 22: 399-411, 2013.

35. Rai V and Agrawal DK: The role of damage- and pathogen-associated molecular patterns in inflammation-mediated vulnerability of atherosclerotic plaques. Can J Physiol Pharmacol 95: 1245-1253, 2017.

36. Jain $M, W u$ B, Pisapia $D$, Salvatore $S$, Mukherjee $S$ and Narula $N$ : A component-by-component characterisation of high-risk atherosclerotic plaques by multiphoton microscopic imaging. J Microsc 268: 39-44, 2017.

37. Ulrich V, Rotllan N, Araldi E, Luciano A, Skroblin P, Abonnenc M, Perrotta P, Yin X, Bauer A, Leslie KL, et al: Chronic miR-29 antagonism promotes favorable plaque remodeling in atherosclerotic mice. EMBO Mol Med 8: 643-653, 2016.

38. Eilenberg W, Stojkovic S, Kaider A, Kozakowski N, Domenig CM, Burghuber C, Nanobachvili J, Huber K, Klinger M, Neumayer C, et al: NGAL and MMP-9/NGAL as biomarkers of plaque vulnerability and targets of statins in patients with carotid atherosclerosis. Clin Chem Lab Med 56: 147-156, 2017.

39. Visse R and Nagase H: Matrix metalloproteinases and tissue inhibitors of metalloproteinases: Structure, function, and biochemistry. Circ Res 92: 827-839, 2003. 
40. Prebble H, Cross S, Marks E, Healy J, Searle E, Aamir R, Butler A, Roake J, Hock B, Anderson N and Gieseg SP: Induced macrophage activation in live excised atherosclerotic plaque. Immunobiology 223: 526-535, 2018.

41. Chen Q, Jin M, Yang F, Zhu J, Xiao Q and Zhang L: Matrix metalloproteinases: Inflammatory regulators of cell behaviors in vascular formation and remodeling. Mediators Inflamm 2013: 928315, 2013.

42. Ohkawara H, Ikeda K, Ogawa K and Takeishi Y: Membrane Type 1-matrix metalloproteinase (Mt1-Mmp) identified as a multifunctional regulator of vascular responses. Fukushima J Med Sci 61: 91-100, 2015

43. Korol RM, Canham PB, Liu L, Viswanathan K, Ferguson GG, Hammond RR, Finlay HM, Baker HV, Lopez C and Lucas AR Detection of altered extracellular matrix in surface layers of unstable carotid plaque: An optical spectroscopy, birefringence and microarray genetic analysis. Photochem Photobiol 87: 1164-1172, 2011.

44. Rohwedder I, Montanez E, Beckmann K, Bengtsson E, Dunér P, Nilsson J, Soehnlein O and Fässler R: Plasma fibronectin deficiency impedes atherosclerosis progression and fibrous cap formation. EMBO Mol Med 4: 564-576, 2012.

45. Newby AC: Metalloproteinases promote plaque rupture and myocardial infarction: A persuasive concept waiting for clinical translation. Matrix Biol 44-46: 157-166, 2015.

46. Starr AE, Bellac CL, Dufour A, Goebeler V and Overall CM: Biochemical characterization and $\mathrm{N}$-terminomics analysis of leukolysin, the membrane-type 6 matrix metalloprotease (MMP25): Chemokine and vimentin cleavages enhance cell migration and macrophage phagocytic activities. J Biol Chem 287: 13382-13395, 2012.

47. Michel JB, Martin-Ventura JL, Nicoletti A and Ho-Tin-Noé B: Pathology of human plaque vulnerability: Mechanisms and consequences of intraplaque haemorrhages. Atherosclerosis 234: 311-319, 2014.

48. Gopalakrishnan M, Silva-Palacios F, Taytawat P, Pant R and Klein L: Role of inflammatory mediators in the pathogenesis of plaque rupture. J Invasive Cardiol 26: 484-492, 2014.

49. Golledge J, McCann M, Mangan S, Lam A and Karan M: Osteoprotegerin and osteopontin are expressed at high concentrations within symptomatic carotid atherosclerosis. Stroke 35: 1636-1641, 2004.
50. Farrokhi E, Samani KG and Chaleshtori MH: Oxidized low-density lipoprotein increases bone sialoprotein expression in vascular smooth muscle cells via runt-related transcription factor 2. Am J Med Sci 349: 240-243, 2015.

51. Fedarko NS, Jain A, Karadag A and Fisher LW: Three small integrin binding ligand $\mathrm{N}$-linked glycoproteins (SIBLINGs) bind and activate specific matrix metalloproteinases. FASEB J 18: 734-736, 2004.

52. Harris NL, Rattray KR, Tye CE, Underhill TM, Somerman MJ, D'Errico JA, Chambers AF, Hunter GK and Goldberg HA: Functional analysis of bone sialoprotein: Identification of the hydroxyapatite-nucleating and cell-binding domains by recombinant peptide expression and site-directed mutagenesis. Bone 27: 795-802, 2000.

53. Yang Y, Cui Q and Sahai N: How does bone sialoprotein promote the nucleation of hydroxyapatite? A molecular dynamics study using model peptides of different conformations. Langmuir 26: 9848-9859, 2010

54. Golledge J, McCann M, Mangan S, Lam A and Karan M: Osteoprotegerin and osteopontin are expressed at high concentrations within symptomatic carotid atherosclerosis. Stroke 35: 1636-1641, 2004.

55. Higgins CL, Isbilir S, Basto P, Chen IY, Vaduganathan M, Vaduganathan P, Reardon MJ, Lawrie G, Peterson L and Morrisett JD: Distribution of alkaline phosphatase, osteopontin, RANK ligand and osteoprotegerin in calcified human carotid atheroma. Protein J 34: 315-328, 2015.

56. Newby AC: Proteinases and plaque rupture: Unblocking the road to translation. Curr Opin Lipidol 25: 358-366, 2014.

57. Newby AC: Newby: Dual role of matrix metalloproteinases (matrixins) in intimal thickening and atherosclerotic plaque rupture. Physiol Rev 85: 1-31, 2005.

58. Steitz SA, Speer MY, McKee MD, Liaw L, Almeida M, Yang H and Giachelli CM: Osteopontin inhibits mineral deposition and promotes regression of ectopic calcification. Am J Pathol 161: 2035-2046, 2002.

59. Gravallese EM: Osteopontin: A bridge between bone and the immune system. J Clin Invest 112: 147-149, 2003.

60. Xu ST, Zou FZ, Cai LN and Xu WL: The downregulation of OPN inhibits proliferation and migration and regulate activation of Erk1/2 in ECA-109 cells. Int J Clin Exp Med 8: 5361-5369, 2015. 